\title{
A New Approach for Estimation of Eigenvalues of Images
}

\author{
Vilas H. Gaidhane \\ ICE Division \\ NSIT, University of Delhi, \\ New Delhi, India
}

\author{
Yogesh V. Hote \\ Indian Institute of Technology \\ (IIT) Roorkee, \\ India
}

\author{
Vijander Singh \\ ICE Division \\ NSIT, University of Delhi, \\ New Delhi, India
}

\begin{abstract}
In this paper, a new approach for estimation of eigenvalues of images is presented. The proposed approach is based on the Gerschgorin's circles theorem. This approach is more efficient as there is no need of calculation of all real eigenvalues. It is also helpful for all type of images where the calculation of eigenvalues may be impractical. More importantly, anyone can come to the conclusion by visual inspection as it is a graphical method. The estimation of eigenvalues can be used to extract the important information of images for various applications.
\end{abstract}

\section{Keywords}

Eigenvalues, Eigenspace, Gerschgorin's theorem, SDDM, SVD, Pattern recognition, Rayleigh quotient.

\section{INTRODUCTION}

The eigenvalues play an important role in image processing applications. There are various methods available for image processing. The processing like measurement of image sharpness can be done using the concept of eigenvalues [1]. Also, the classification of image such as coin and face is done using an eigen-space approach [2]. In this, the eigenvalues are calculated using the singular value decomposition (SVD). In case of human face segmentation using elliptical shape [3], largest and smallest eigenvalue of covariance matrix represent the elliptical shape. The major and minor axial length of an ellipse is depending on the largest and smallest eigenvalue of covariance matrix of an image. Thus, in such image processing applications, one of the most important things about an image is its eigenvalues knowledge. But all these methods require lot of computation and analysis. It is also impossible to describe behavior of eigenvalues by simple inspection. One way of estimating the eigenvalues is singular value decomposition (SVD) of covariance matrix which is used by many researchers in image processing applications. But, this technique demands $O^{3}$ level of calculations which increases the computational complexity of the system [4]. Another method for estimating the eigenvalues is to obtain the graph (trace) of matrix which is generally used in control system applications. It has small trace but very wide range of eigenvalues of a matrix. In literature, Gerschgorin suggested a method which gives bounded region that contain all eigenvalues of a matrix [5] [6]. This theorem also used to find the circles whose union contains all the eigenvalues of complex matrices [7]. Moreover, it has two eigenvalues with opposite sign whose absolute values are large. In this paper, authors proposed a new approach using Gerschgorin's theorem for estimation of eigenvalues and apply it successfully to study the features of an image.

The rest of the paper is structured as follows. The generalized eigenvalues problem in image processing is discussed in section
2. A brief introduction of Gerschgorin's theorem and its utility is presented in section 3. The detailed explanation of proposed approach for estimation of eigenvalues is described in section 4. The experimental results for different images are explained in section 5. Finally, conclusions are drawn in section 6.

\section{GENERALIZED EIGENVALUE PROBLEMS IN IMAGE PROCESSING}

For optimization process, Rayleigh quotient is stated as [8],

$$
\mathcal{R}(\boldsymbol{x})=\max \frac{\boldsymbol{x}^{T} \boldsymbol{P} \boldsymbol{x}}{\boldsymbol{x}^{T} \boldsymbol{Q} \boldsymbol{x}}
$$

where, $\mathrm{P}, \mathrm{Q} \in \mathcal{R}^{n \times n}$ are symmetric matrices and $\boldsymbol{x}$ is nonzero vector. If the constraint is chosen as, $\boldsymbol{x}^{T} Q \boldsymbol{x}=1$, then the constrained optimization problem can be defined in the form as,

$$
\begin{gathered}
P \boldsymbol{x}-\lambda \boldsymbol{Q x}=0 \\
\boldsymbol{P} \boldsymbol{x}=\lambda \boldsymbol{Q x}
\end{gathered}
$$

The optimization value reached by solving eq. (2) is equal to the maximum eigenvalue $\lambda$. This is used for solving the symmetric matrices. The nonsymmetrical eigenvalue problem can be solve by considering the $Q^{-1}$ on the left hand side of eq. (2). Let, $\boldsymbol{Q}=\boldsymbol{A}^{T} \boldsymbol{A}$, where $\boldsymbol{A}$ is nonsingular matrix, the eigenvalue problem in eq. (2) is converted to symmetric eigenvalue problem as [1],

$$
\begin{aligned}
& \boldsymbol{P} \boldsymbol{x}=\lambda \boldsymbol{A}^{T} \boldsymbol{A} \boldsymbol{x} \\
& \boldsymbol{P} \boldsymbol{A}^{-1} \boldsymbol{A} \boldsymbol{x}=\lambda \boldsymbol{A}^{T} \boldsymbol{A x} \\
& P \boldsymbol{A}^{-1} \boldsymbol{v}=\lambda \boldsymbol{A}^{T} \boldsymbol{v} \\
& {\left[\left(A^{-1}\right)^{T} P A^{-1}\right] v=\lambda v}
\end{aligned}
$$

This is the general eigenvalue problem and can be solve for different values of $\lambda$. Let $\boldsymbol{V}=\left[\boldsymbol{v}_{\mathbf{1}}, \ldots, \boldsymbol{v}_{\boldsymbol{n}}\right]$ is the eigenvector with respect to eigenvalue $\lambda$. Here the largest eigenvalue $\lambda_{1}$ represent the direction of maximum variance of the matrix $\boldsymbol{P}$. Most of the image features are shown by the larger eigenvalues whereas smaller eigenvalues are more sensitive to noise. Thus, the reduction of dimension by elimination of small eigenvalues is possible in image processing. This process leads towards simple computation and less complexity. For eigenvalue problem, the matrix $\boldsymbol{A}$ can be decomposed using the Singular Value Decomposition (SVD) given as [1],

$$
\boldsymbol{A}=\boldsymbol{U} \boldsymbol{\Sigma} \boldsymbol{V}^{T}
$$

where, $\boldsymbol{U}=\left\{u_{1}, u_{2}, \ldots, u_{n}\right\}$ is the orthogonal vectors, $\boldsymbol{V}=$ $\left\{v_{1}, v_{2}, \ldots, v_{n}\right\}$ is the set of orthogonal eigenvectors and the diagonal elements of $\boldsymbol{\Sigma}=\left\{\sigma_{1}, \sigma_{1}, \ldots, \sigma_{n}\right\}$ are real and positive singular values representing the eigenvalues $\lambda_{i}=\sigma_{i}^{2}$. In such 
type of eigenvalues and eigenvector calculations, $O^{3}$ levels of operations are required which increases computational time. Here, authors proposed a simple graphical approach using the Gerschgorin's theorem to estimate the eigenvalues of images. The proposed approach overcomes the eigenvalues problem occur due to singular value decomposition (SVD).

\section{GERSCHGORIN'S THEOREM}

Before Gerschgorin's theorem, it is necessary to introduce the form of matrix to solve the theorem commonly known as Strictly Diagonally Dominant Matrix (SDDM). The condition for SDDM is defined as [9]:

$$
\left|x_{i i}\right|>\sum_{j \neq i}\left|x_{i j}\right| \text { for } i=1,2, \ldots, m .
$$

If this condition is satisfied then the matrix $\boldsymbol{X}$ is always nonsingular. By knowing the matrix $\boldsymbol{X}$ is nonsingular, Gerschgorin's theorem can be stated. Given a Complex square matrix $\boldsymbol{X}=\left[x_{i j}\right]$, where $i=j=1,2, \ldots, n$ and $n \geq 2$. In this case, the eigenvalues of $\boldsymbol{X}$ is similar to the zeros of the transfer function polynomial as:

$$
\boldsymbol{T}(s)=|\{s I-\boldsymbol{X}\}|
$$

Where, $I$ is the identity matrix of order $n \times n$. But in image applications, $n$ is very and therefore it is not easy to estimate the eigenvalues. In 1931, Russian Mathematician S. Gerschgorin [5] developed a new technique to estimate the eigenvalues known as 'Gerschgorin's Theorem' which states that if the sum of moduli of element along the $i^{t h}$ row excluding the diagonal element $x_{i i}$ is less than the diagonal element $x_{i i}$ then every eigenvalue of $\boldsymbol{X}$ matrix lies inside or on the boundary of circles.

$$
\left|\lambda-x_{i i}\right| \leq \sum_{j \neq i}\left|x_{i j}\right| \quad \text { for } i=\{1,2,3, \ldots, n\}
$$

Where, $\lambda$ is an eigenvalue of matrix $\boldsymbol{X}$. Gerschgorin closed complex $i^{\text {th }}$ circle of the matrix $\boldsymbol{X}$ is given as:

$$
D_{i} \triangleq\left\{z \in \mathbb{C}:\left|z-x_{i i}\right| \leq\left|d_{i}\right|\right\}
$$

The Gerschgorin circle is the interior region and the boundary of the circle having $x_{i i}$ as center of the circle. The circle has a radius $d_{i}$ is defined as:

$$
d_{i}=\sum_{j \neq 1}\left|x_{i j}\right|
$$

The center of the Gerschgorin circle is real part of the matrix $\boldsymbol{X}$, i.e., $x_{i i}$. The above results are true for each eigenvalue $\lambda$ of matrix $\boldsymbol{X}$. If $\lambda(\boldsymbol{X})$ denotes the set of all eigenvalues, then

$$
\lambda(X) \subseteq \bigcup_{i=1}^{n} D_{i}
$$

In eq. (10), $\lambda$ is the eigenvalue of the matrix $\boldsymbol{X}$ and there is a complex eigenvector $\boldsymbol{y}=\left[y_{1}, y_{2}, \ldots, y_{n}\right]^{T}$, with $\boldsymbol{y} \neq 0$, such that $\boldsymbol{X} \boldsymbol{y}=\lambda \boldsymbol{y}$. As $\neq 0, i^{\text {th }}$ component of $\boldsymbol{X} \boldsymbol{y}=\lambda \boldsymbol{y}$ results into the equation as:

$$
\begin{gathered}
\sum_{j=1}^{n}\left(x_{i, j}\right) y_{j}=\lambda \boldsymbol{y} \quad \text { or } \\
\sum_{\substack{j=1 \\
j \neq i}}^{n}\left(x_{i, j}\right) y_{j}=\left(\lambda-x_{i, i}\right) y_{i}
\end{gathered}
$$

By considering the absolute values in eq. (11) and triangle inequality, gives,

$$
\left|\lambda-x_{i, i}\right|\left|y_{i}\right| \leq \sum_{\substack{j=1 \\ j \neq i}}^{n}\left|\left(x_{i, j}\right)\right|\left|y_{j}\right|
$$

From above equation $\left|y_{j}\right| \leq\left|y_{i}\right|$ for all $1 \leq j \leq n$. Dividing eq. (12) by $\left|y_{i}\right|>0$, gives the result $\lambda \in \boldsymbol{D}_{i}$. The intersection of Gerschgorin circles gives the range of bound under which the eigenvalues exist. Such bound of eigenvalues is the extreme ends of the intersection of the circles which is known as Gerschgorin bound. The Gerschgorin circle theorem and estimation of eigenvalues is explained using the different examples in section 3.1, 3.2 and 3.3.

\subsection{Gerschgorin Circle Example 1}

Consider a $2 \times 2$ matrix $X$ as [6],

$$
X=\left[\begin{array}{cc}
1 & 3 \\
2 & -3
\end{array}\right]
$$

The matrix $X$ has the eigenvalues 2.1623 and -4.1623 . Therefore, the matrix $X$ has two circles, first with radius 3 centered at $(1,0)$ and second circle is of radius 2 and centered at $(-3,0)$. The plot of Gerschgorin circles and eigenvalues in complex plane is shown in Figure 1. According to the Gerschgorin circle theorem, every eigenvalue must lie within one of the circle. The both eigenvalues may lie either in one of the circle or one in each circle.

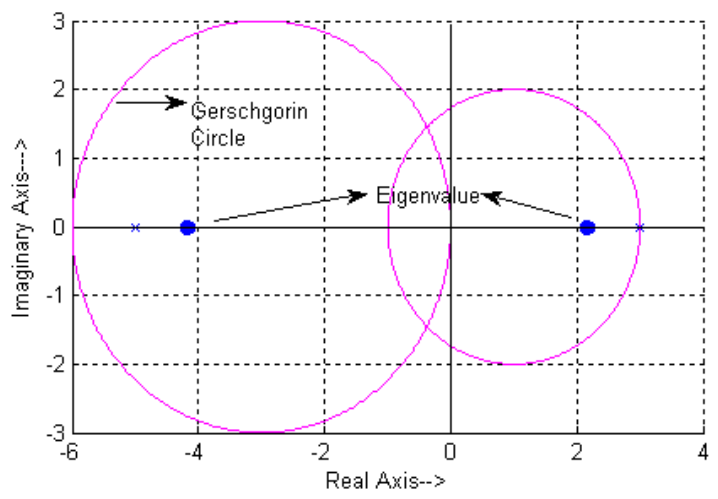

Fig 1: Gerschgorin's circles and eigenvalues

\subsection{Gerschgorin Circle Example 2}

Consider another $2 \times 2$ matrix $\boldsymbol{X}$ as [6] [7],

$$
\boldsymbol{X}=\left[\begin{array}{ll}
2 & -2 \\
4 & -2
\end{array}\right]
$$

The matrix $\boldsymbol{X}$ has the eigenvalues $2 i$ and $-2 i$. The matrix $\boldsymbol{X}$ has two circles, first with radius 2 centered at $(2,0)$ and second circle of radius 4 and centered at $(-2,0)$. The plotting of both Gerschgorin circles and the eigenvalues in the complex plane is shown in Figure 2. It is observed from Figure 2 that the eigenvalues of matrix $\boldsymbol{X}$ fall under the circle of $2^{\text {nd }}$ row of matrix $\boldsymbol{X}$ and none of the eigenvalue fall under the circle of $1^{\text {st }}$ row. It is also observed from Figure 1 and Figure 2 that the eigenvalues may be real or complex.

\subsection{Gerschgorin's Circle Example 3}

Consider the $2 \times 2$ matrix $\boldsymbol{X}$ as [6] [7],

$$
\boldsymbol{X}=\left[\begin{array}{ll}
3 & -2 \\
4 & -2
\end{array}\right]
$$

The matrix $\boldsymbol{X}$ has complex eigenvalue, $0.5000+1.3229 i$ and $0.5000-1.3229 i$. The matrix $\boldsymbol{X}$ has two circles, first with radius 2 centered at $(3,0)$ and second circle of radius 4 and centered at $(-2,0)$. The transpose of matrix $\boldsymbol{X}$ is represented by $\boldsymbol{X}^{T}$ which is equivalent to column matrix $\boldsymbol{X}$. The eigenvalues of $\boldsymbol{X}$ are same as the eigenvalues of $\boldsymbol{X}^{T}$. As the rows of $\boldsymbol{X}^{T}$ 
correspond to the column of $\boldsymbol{X}$, the eigenvalues lie inside Gerschgorin circle corresponding to the column of $\boldsymbol{X}$.

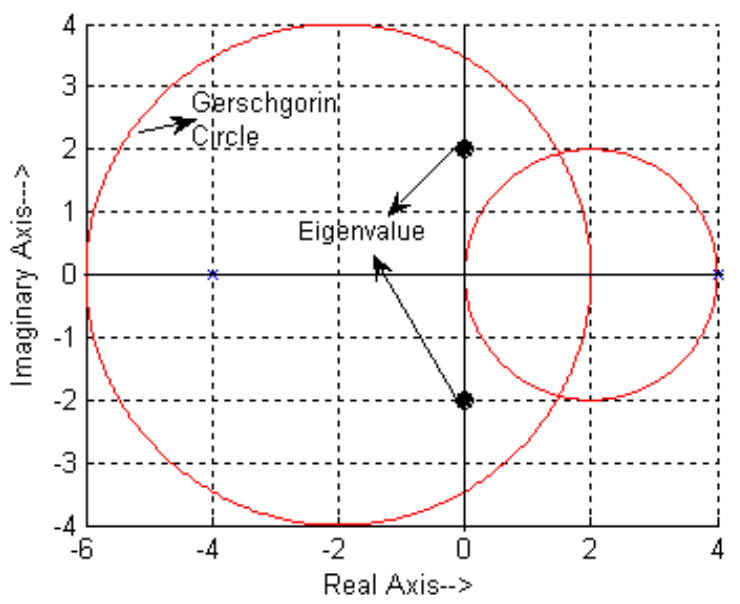

Fig 2: Gerschgorin circles and eigenvalues

Figure 3 shows the Gerschgorin circles and eigenvalues of $\boldsymbol{X}$ and $\boldsymbol{X}^{T}$. From above examples it is clear that the Gerschgorin theorem can be used to estimate eigenvalues of any type of matrices.

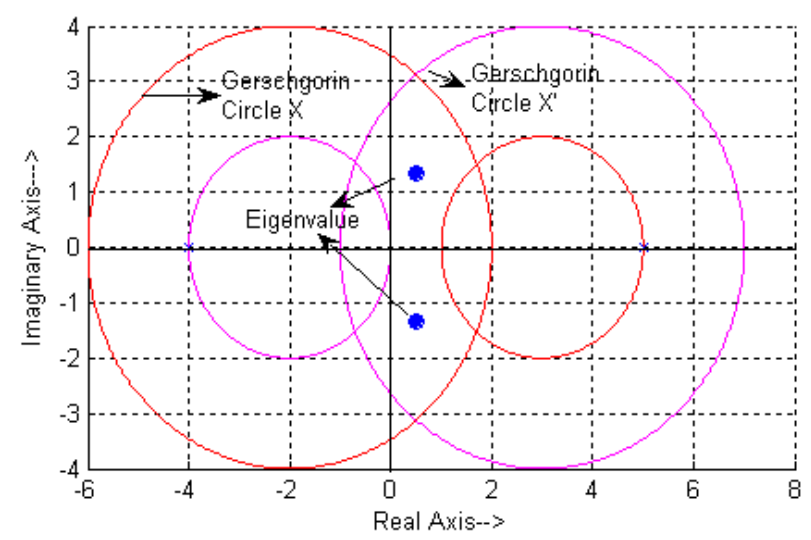

Fig 3: Gerschgorin circles and eigenvalues

\section{PROPOSED APPROACH FOR ESTIMATION OF EIGENVALUES OF IMAGES}

An image is considered to be a function of two real variables $x$ and $y$ with function $f(x, y)$ and magnitude ranges from 0 to 255. The 2D image represented by $f(x, y)$ is divided into $N$ rows and $M$ columns. The eigenvalues of such $N$ rows and $M$ columns matrix shows the main feature of the images. In image processing techniques such as image enhancement, image compression [10], pattern recognition [11] and face identification [12], calculation of eigenvalues and eigenvectors is required. The largest eigenvalue represent the important (dominant) features of the image whereas the smallest eigenvalue represent the less important features or noise which can be neglected. The Gerschgorin's theorem explained in above section is useful to estimate the eigenvalues of image. In this paper, authors proposed a new approach for statistical and graphical estimation of eigenvalues using Gerschgorin's theorem concept. The proposed approach is described in the following steps:

Step 1: Obtain 2D gray scale image $f(x, y)$.

Step 2: Construct the gray image $f(x, y)$ in the form of $N$ rows and $M$ columns matrix.

Step 3: Obtained the image vector $\Gamma_{i}$ using the chucks of $4 \times$ $4,8 \times 8,16 \times 16, \ldots, n \times n$, (where, $n=2,4,16,32,64, \ldots)$. The image vector $\boldsymbol{\Gamma}_{i}$ should be of order $n^{2} \times M$.

Step 4: Compute the average vector:

$$
\boldsymbol{\psi}=\frac{1}{M} \sum_{i=1}^{M} \Gamma_{i}
$$

Step 5: Subtract the average vector:

$$
\boldsymbol{\sigma}_{i}=\Gamma_{i}-\boldsymbol{\psi}
$$

Step 6: Obtain the covariance matrix $\boldsymbol{C}$,i.e.,

$$
\boldsymbol{C}=\frac{1}{M} \sum_{i=1}^{M}\left(\boldsymbol{\sigma}_{i}^{T} \boldsymbol{\sigma}_{i}\right)=\boldsymbol{A}^{T} \boldsymbol{A}
$$

where, $\boldsymbol{A}=\left\{\boldsymbol{\sigma}_{1}, \boldsymbol{\sigma}_{2}, \ldots, \boldsymbol{\sigma}_{M}\right\}$ is the $M \times M$ matrix.

Step 7: Apply the Gerschgorin's theorem stated in section 3 and draw Gerschgorin's circles,

$$
\boldsymbol{D}_{i} \triangleq\left\{z \in \mathbb{C}:\left|z-x_{i i}\right| \leq\left|d_{i}\right|\right\}
$$

Step 8: Analyze the graph and estimate the eigenvalues using,

$$
\lambda(\boldsymbol{X}) \subseteq \bigcup_{i=1}^{n} \boldsymbol{D}_{i}
$$

Proposed comment: From observations on Gerschgorin circles, heuristically, largest eigenvalue of the image can be written as,

$$
\lambda_{\max } \cong \frac{|D|+|E|}{2}
$$

where, $\mathrm{D}$ is the extreme left bound and $\mathrm{E}$ is the extreme right bound. The value of $\lambda_{\min }$ is very small as compared to $\lambda_{\max }$ and it is near to origin.

In proposed algorithm, covariance matrix reduces the dimension of image matrix and it considers only important features of an image. The use of covariance matrix reduces calculation complexity and time [13] [14]. The proposed approach using Gerschgorin theorem gives the range of eigenvalues using the bound region and the nature of features of an image graphically. This approach is very simple and it can be used in image processing applications such human face identification, pattern recognition and similarity measure etc. The proposed approach using Gerschgorin's theorem is applied on different images and explained in next section.

\section{EXPERIMENTAL RESULTS AND ANALYSIS}

For experiments, authors considered different colored images shown in Figure 4. The set of images (Figure 4a, b, c and e) are collected by authors with digital camera and formatted by Adobe Photoshop 7.0.1 software. The set of images (Figure 4d, $\mathrm{f}, \mathrm{g}$ and $\mathrm{h}$ ) are collected from CVG-UGR-Image database. The images are cropped into $256 \times 256$ pixel (Figure 4a, b, c, d) and $128 \times 128$ (Figure 4e, f, g, h) pixel size. Since, in proposed approach authors have used the concept of covariance matrix, the number of eigenvalues totally depends on the size of covariance matrix. 


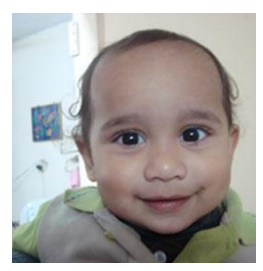

(a) Mayank

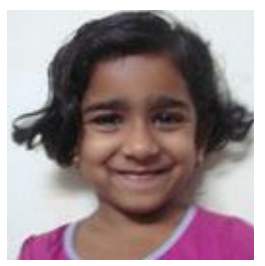

(e) Mrudula

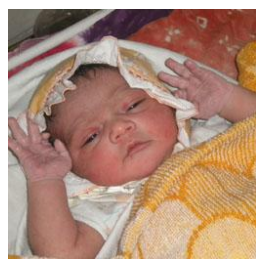

(b) Manu

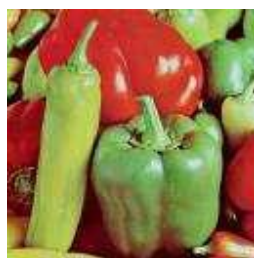

(f) Peppers

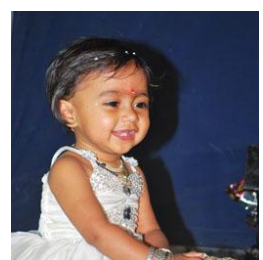

(c) Manasvi

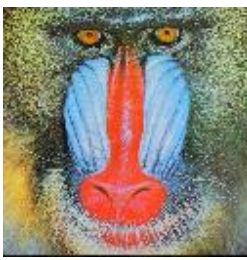

(g) Baboon

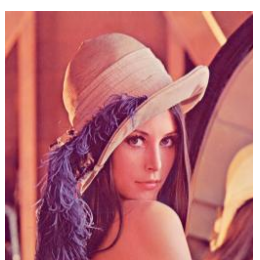

(d) Leena

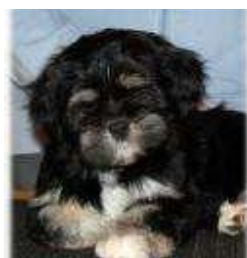

(h) doggy

Fig 4: Input Images used for experiments i) a, b, c, d $256 \times 256$ pixel images

ii) e, f, g, h $128 \times 128$ pixel images
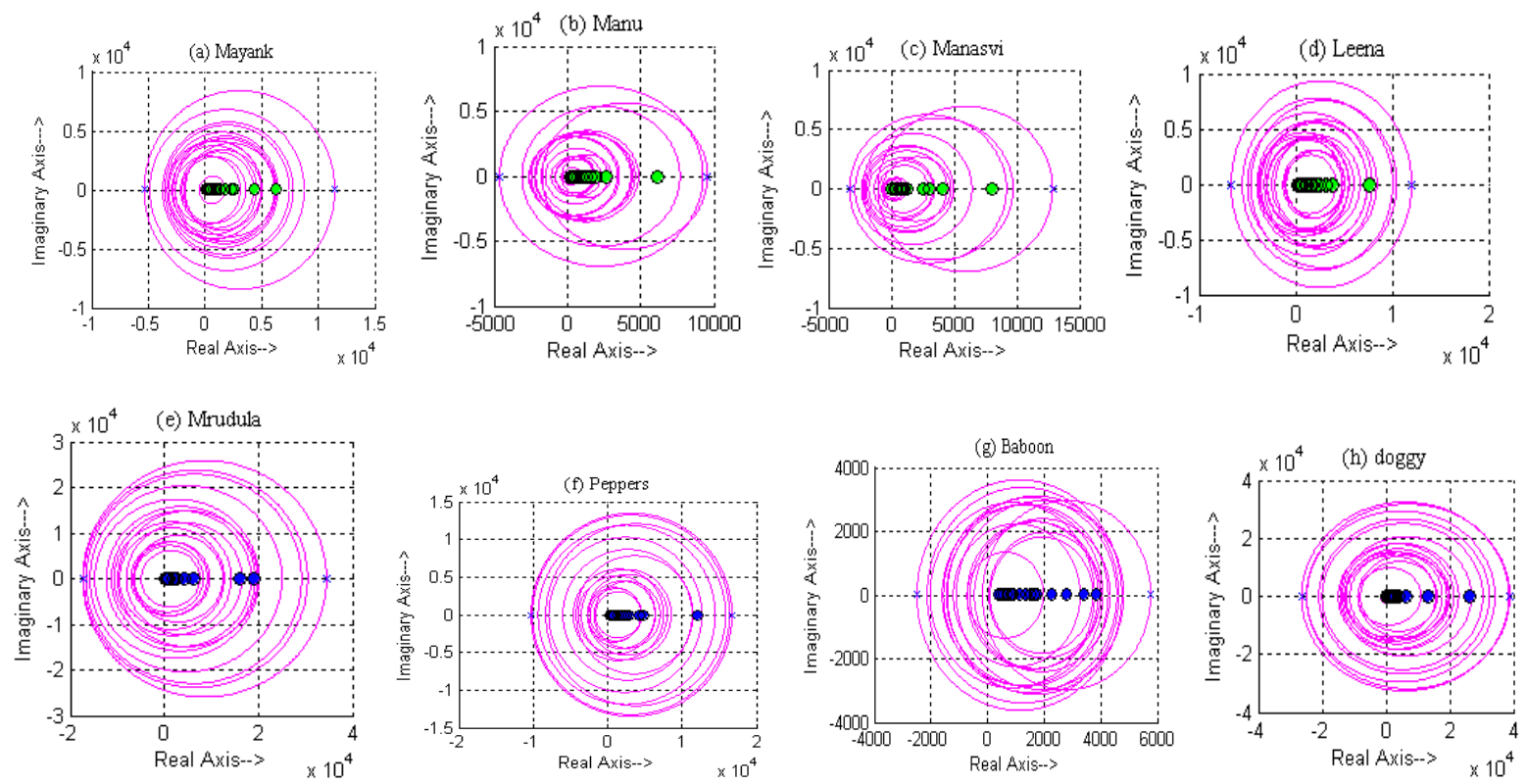

Fig 5: Gerschgorin circles and left and right bound of images 
Table 1. Left and Right Bound, Smallest and largest eigenvalue of different images obtained using proposed approach (all values are multiplied by $10^{4}$ )

\begin{tabular}{|l|c|c|c|c|}
\hline \multicolumn{1}{|c|}{$\begin{array}{c}\text { Input } \\
\text { Image }\end{array}$} & $\begin{array}{c}\text { Left } \\
\text { Bound }\end{array}$ & $\begin{array}{c}\text { Right } \\
\text { Bound }\end{array}$ & $\begin{array}{c}\text { Smallest } \\
\text { Eigenvalue }\end{array}$ & $\begin{array}{c}\text { Largest } \\
\text { Eigenvalue }\end{array}$ \\
\hline (a)Mayank & -0.5 & 1.1 & 0.04 & 0.761 \\
\hline (b) Manu & -0.4 & 0.81 & 0.019 & 0.64 \\
\hline (c)Manasvi & -0.25 & 1.3 & 0.0007 & 0.78 \\
\hline (d)Leena & -0.6 & 1.1 & 0.167 & 0.85 \\
\hline (e)Mrudula & -1.8 & 3.5 & 0.0191 & 2.1132 \\
\hline (f)Peppers & -1 & 1.6 & 0.0283 & 1.3066 \\
\hline (g)Baboon & -0.22 & 0.6 & 0.003 & 0.408 \\
\hline (h)doggy & -2.2 & 3.8 & 0.0228 & 2.844 \\
\hline
\end{tabular}

Table 2. Computational time (second) required to estimate the eigenvalues using SVD and proposed approach

\begin{tabular}{|l|c|c|c|}
\hline \multicolumn{1}{|c|}{$\begin{array}{c}\text { Input } \\
\text { Image }\end{array}$} & $\begin{array}{c}\text { Image Size } \\
\text { (Pixel) }\end{array}$ & $\begin{array}{c}\text { Computati } \\
\text { onal Time } \\
\text { using SVD } \\
\text { (second) }\end{array}$ & $\begin{array}{c}\text { Computational } \\
\text { Time using } \\
\text { Proposed approach } \\
\text { (second) }\end{array}$ \\
\hline (a)Mayank & $256 \times 256$ & 0.472942 & 0.369934 \\
\hline (b) Manu & $256 \times 256$ & 0.399273 & 0.270651 \\
\hline (c)Manasvi & $256 \times 256$ & 0.417301 & 0.290164 \\
\hline (d)Leena & $256 \times 256$ & 0.426137 & 0.298381 \\
\hline (e)Mrudula & $128 \times 128$ & 0.264216 & 0.201914 \\
\hline (f)Peppers & $128 \times 128$ & 0.274758 & 0.207521 \\
\hline (g)Baboon & $128 \times 128$ & 0.275741 & 0.189025 \\
\hline (h)doggy & $128 \times 128$ & 0.289467 & 0.180168 \\
\hline
\end{tabular}

It is a squared matrix of size $16 \times 16,32 \times 32,64 \times 64, \ldots, M \times$ $M$ pixel. Where, maximum value of $M$ is depending on the size of image. For $256 \times 256$ image size, $M$ may be 16 , $32,64,128$ or 256 (maximum $M=256$ ). It is observed from Figure 5 that features of image '(a) Mayank' is smoothly distributed as compared to image '(b) Manu'. This is due to the fact that the variation of colors inside the image '(a) Mayank' are very less. In this, the variation in Gerschgorin circles is smooth. Similarly, the features of image ' $(\mathrm{g})$ Baboon' are more diverged as compared to ' $(\mathrm{h})$ doggy'. This is due to the reason that the variation of colors inside the image ' $(\mathrm{g})$ Baboon' is very large. Here, the radius of Gerschgorin circle is changing abruptly. It is also observed that the divergence of the Gerschgorin circles is more wherever the variation in the features of images is large. Table 1 represents the largest and smallest eigenvalue of different images obtained using the proposed approach. The largest eigenvalue of $256 \times 256$ pixel image '(a) Mayank' is $0.761 \times 10^{4}$ and smallest $0.04 \times 10^{4}$. Similarly, the largest eigenvalue of $128 \times 128$ pixel image ' $(g)$
Baboon' is $0.408 \times 10^{4}$ and smallest eigenvalue is $0.003 \times$ $10^{4}$. Table 2 shows the time required to estimate the eigenvalue using the SVD technique and proposed approach. It is observed that the proposed approach needs less time as compared to techniques using the SVD decomposition. Thus, the proposed approach is more accurate and efficient.

\section{CONCLUSION}

A new approach using the concept of Gerschgorin's theorem for estimation of eigenvalues of an image is proposed. This is the new concept in the field of image processing which can be used for any size of images. The proposed approach is applied on different size of images. The computational time and complexity for estimating the eigenvalues is reduced. It is concluded that the proposed approach is very simple and effective to analyze the different size of images.

\section{REFERENCES}

[1] Wee, C. Y. and Paramesran, R. 2008. Image Sharpness measure using Eigenvalues. In Proceeding of IEEE $9^{\text {th }}$ international conference on Signal processing, ICSP2008, Beijing, pp. 840-843.

[2] Huber, R., Ramoser, H., Mayer, K., Penz, H. and Rubik, M. 2005. Classification of coin using an eigenspace approach. Pattern Recognition Letters, Elsevier, vol. 26(12), pp. 6175.

[3] Adipranata, R., Cherry, E., Ballangan, G. and Ongkodjojo, R. P. 2009. Fast Method for multiple Human Face Segmentation in color Image. International journal of Advanced Science and Technology, vol. 3, pp. 19-32.

[4] Wee, C. Y. and Paramesran, R. 2006. Measure of image sharpness using eigenvalues. An international journal of Information Sciences, Elsevier, vol. 177(12), pp. 25332552.

[5] Gerschgorin, S. 1931. Ueber die Abgrenzung der Eigenwerte einer Matrix. Izv. Akad. Nauk. SSSR Ser. Mat., vol. 1, pp. 749-754.

[6] Hote, Y. V., Choudhury, D. R. and Gupta, J. R. P. 2006. Gerschgorin Theorem and its applications in Control System Problems. IEEE Conference on Industrial Electronics, pp. 2438-2443.

[7] Hote, Y. V. 2010. A New Method of determining Instability of Linear System. In proceeding of International Conference on Advances in Electrical and Electronics, pp. 132-136.

[8] Strang, G. 1980. Linear Algebra and Its Applications. Second edition, Academic Press, New York.

[9] Gaidahne, V., Singh, V. and Kumar, M. 2010. Image Compression using PCA and Improved Technique with MLP Neural Network. In Proceeding of IEEE international conference on Advances in Recent Technologies in Communication and Computing, pp.106-110.

[10] Ranade, A., Mahabalarao, S. S. and Kale, S. 2007. A variation on SVD based image compression. J. of Image and Vision Computing, vol. 25, pp. 771-777. 
[11] Sun, T. H., Liu C. S. and Tien, F. C. 2008. Invariant 2D objects recognition using eigenvalues of covariance matrices, re-sampling and autocorrelation. Expert System with Applications, vol. 35, pp. 1966-1977.

[12] Xu, Y., Song, F., Feng, G. and Zhao, Y. 2010. A novel local preserving projection scheme for use with recognition. Expert Systems with Applications, vol. 37(9), pp. 6718-6721.

[13] Li, B. and Tsatsomeros, M. J. 1997. Doubly Diagonally Dominant Matrices. Linear Algebra and its applications, Elsevier Science, pp. 221-235.

[14] Gaidahne, V., Singh, V., Hote, Y. V. and Kumar, M. 2011 New Approaches for Image Compression using Neural Network. Journal of Intelligent Learning Systems and Applications. (Accepted and in Press).

\section{AUTHORS PROFILE}

Vilas H. Gaidhane received his B.E degree in Electronics Engineering from Nagpur University, Nagpur in 2000. Then, he received M.Tech. degree in VLSI Design, from UP Technical University, Lucknow, in 2010. He is pursuing Ph. D degree in Instrumentation and Control Engineering Division in Netaji
Subhas Institute of Technology, New Delhi, affiliated to University of Delhi, Delhi. His field of research includes Robust Control, Artificial Intelligence and Image Processing.

Yogesh V. Hote received his B.E. degree in Electrical Engineering from Govt. college of Engineering, Amravati, in 1998. Then, he received M.E. degree in Control Systems, from Govt. college of Engineering, Pune, in 2000.

He also received $\mathrm{Ph}$. D degree in Instrumentation and Control Engineering, University of Delhi in 2009. His field of research includes robust control, robotics, numerical analysis and power electronics. He is working as Assistant Professor in Electrical Engineering Department, Indian Institute of Technology (I.I.T.) Roorkee, India.

Vijander Singh received his B. Tech in Electrical Engineering from G.B. Pant University of Agriculture and Technology, Uttarakhand in 1995. He received M.E. degree in Electrical Engineering in 2000 and Ph. D in Electrical Engineering in 2007 from IIT Roorkee. He is working as Associate Professor in Instrumentation and Control Engineering Division in Netaji Subhas Institute of Technology, New Delhi, affiliated to University of Delhi, Delhi. His areas of research are Process control, Biomedical Instrumentation, Artificial Intelligence and Image Processing. 